\title{
Spatio-temporal difference analysis of land use efficiency in Lanxi Urban Agglomeration based on SBM-Undesirable model
}

\author{
Chunyu Fu, Peiji Shi, Tao Feng, and Weiping Zhang \\ College of Geography and Environment Science,Northwest Normal University,Lanzhou 730000,China
}

\begin{abstract}
Based on the connotation of green development, SBM-Undesirable model was used to measure the land use efficiency of Lanxi Urban Agglomeration in 2006, 2010, 2014 and 2018, and Theil Index and Spatial Autocorrelation Methods were used to reveal the spatial difference characteristics. The results show that: (1) From 2006 to 2018, the county land use efficiency of Lanxi Urban Agglomeration increased steadily, and the regional differences first expanded and then narrowed. (2) The land use efficiency of counties in Lanxi Urban Agglomeration presents a spatial difference law of "high in the middle-low in the periphery, high in the west and low in the east, and differentiated between the north and the south", while the two provinces present a decreasing law of "west-east". (3) The spatial agglomeration characteristics of land use efficiency in Lanxi Urban Agglomeration are remarkable. Locally, the high-high agglomeration is typical in the central region, while the low-low agglomeration spreads from southeast to southwest.
\end{abstract}

\section{Introduction}

Land is an important natural resource carrying the production and living activities of human society. Green and efficient use of land is also an indispensable prerequisite for sustainable development of social economy. With the advancement of industrialization and urbanization, the contradiction between socioeconomic development and resource environment has gradually become prominent, resulting in serious problems such as unreasonable internal structure of construction land, inefficient land use, high resource consumption and high pollution emission. China puts forward the concept of green development in the 13th Five-Year Plan, which shows that realizing green development is an inevitable choice to alleviate the current contradictions ${ }^{[1]}$. The concept of green development emphasizes ecological protection and sustainable development in the process of land use.That is, the maximization of economic benefits, social benefits,ecological and environmental benefits and the minimization of negative environmental effects of land use, and the realization of green land use methods.

Foreign' research on land use efficiency focuses on the measurement model and system construction of land use efficiency, realistic mechanism and influencing factors,optimization policy measures ${ }^{[2-3]}$. On the basis of relevant theories and the actual situation in China, domestic scholars have conducted in-depth research on land use efficiency evaluation and analysis ${ }^{[4-5]}$, influencing factors ${ }^{[6-7]}$, and coupling relationship with urbanization and industrial integration ${ }^{[8]}$. The traditional land use efficiency model pays more attention to the expected output in the process of land use, without considering the problems of environmental degradation and excessive consumption of resources in the actual process of land development. With the deepening of ecological civilization construction in China, the connotation of land use efficiency has also changed. In recent years, many scholars began to pay attention to the influence of environmental factors on land use process, and studied the relationship between land use and green development, the specific indicators of environmental factors and how to incorporate them into the evaluation system, so as to explore ways and strategies to improve land use efficiency.

The green development index evaluation system is used for reference, the environmental factors are included in the index system to measure the land use efficiency of 39 counties in Lanxi Urban Agglomeration from 2006 to 2018, and the spatial and temporal differences of land use efficiency are explored from multiple scales with the help of Theil Index and spatial autocorrelation. The results will provide scientific reference for the rational allocation of land use structure, ecological protection and the formulation of relevant policies and measures in Lanxi urban agglomeration.

\section{Study area, method and index system}

\subsection{Study area}

Lanxi Urban Agglomeration is an important interprovincial urban agglomeration in western China. This paper takes the Lanzhou-Xining urban agglomeration (Table 1) as the study area, including 39 county units.

Table 1. Research area scope

\footnotetext{
* Corresponding author: fuchunyu96@163.com
} 


\begin{tabular}{|c|c|c|}
\hline Province & $\begin{array}{l}\text { City and } \\
\text { state }\end{array}$ & Counties (districts and cities) \\
\hline \multirow{4}{*}{ Gansu } & Lanzhou & $\begin{array}{c}\text { Chengguan, Qilihe, Xigu, Anning, } \\
\text { Honggu, Yongdeng, Yuzhong, } \\
\text { Gaolan }\end{array}$ \\
\hline & Baiyin & Baiyin, Pingchuan, Jingyuan,Jingtai \\
\hline & Dingxi & Anding, Longxi, Weiyuan,Lintao \\
\hline & Linxia & $\begin{array}{c}\text { Linxia, dongxiang, Yongjing, } \\
\text { Jishishan }\end{array}$ \\
\hline \multirow{5}{*}{ Qinghai } & Xining & $\begin{array}{c}\text { Chengdong, Chengzhong, Chengxi, } \\
\text { Chengbei, Datong, } \\
\text { Huangzhong, Huangyuan }\end{array}$ \\
\hline & Haidong & $\begin{array}{c}\text { Ledu, Ping 'an, Huzhu, Minhe, } \\
\text { Hualong, Xunhua }\end{array}$ \\
\hline & Haibei & Haiyan \\
\hline & Hainan & Gonghe, Guide, Guinan \\
\hline & Huangnan & Tongren, Jianzha \\
\hline
\end{tabular}

\subsection{Research method}

\subsubsection{SBM-Undesirable model}

The disadvantage of traditional DEA model is that it does not consider the slack of variables, and the measurement results are inaccurate. Therefore, the SBMUndesirable model considering environmental variables is chosen here, which not only solves the slack of inputoutput variables, but also takes into account the efficiency evaluation of unexpected outputs ${ }^{[9]}$.

\subsubsection{Theil Index}

Theil Index is used to measure the difference of a certain attribute in the study area, and to explore the change of differences between regions and within regions in the decomposition of total differences between regions ${ }^{[10]}$.

\subsubsection{Spatial autocorrelation}

In order to determine whether there is agglomeration or heterogeneity in the spatial relationship of land use efficiency in adjacent or adjacent counties, Moran's I index in Spatial Autocorrelation analysis method is used to measure and test $\mathrm{it}^{[11]}$.

\subsection{Index system construction}

According to the basic principle of SBM-Undesirable model, considering the availability of data and the representativeness of indicators, the index system of land use efficiency is constructed from three aspects: input, expected output and unexpected output.

Land, capital and labor input are measured by the built-up area of the county, the investment in fixed assets and the number of employees per unit. The economic, social and environmental benefits of the expected output indicators are characterized by the added value of the secondary and tertiary industries, the total sales of social consumer goods and the green space coverage rate of the built-up areas in the county. Due to the small proportion of pollutants discharged from the tertiary industry, the industrial wastewater discharge and industrial sulfur dioxide discharge are mainly used to characterize the undesirable output.

\section{Results and analysis}

\subsection{Time series change characteristics of county land use efficiency}

Based on SBM-Undesirable model, the land use efficiency of 39 county units in Lanxi Urban Agglomeration from 2006 to 2018 was calculated, and the average land use efficiency of Lanxi Urban Agglomeration was obtained after data processing. During the study period, the land use efficiency (Table 2) of counties in Lanxi Urban Agglomeration increased on the whole, and Theil Index showed a "rising-falling" inverted "V" trend, reaching the highest value in 2010. The results show that there are two stages in the evolution of land use efficiency in Lanxi Urban Agglomeration. The first stage (2006-2010): the land use efficiency increased from 0.4995 in 2006 to 0.5484 in 2010 , with an average annual growth rate of $1.96 \%$; Theil Index increased from 0.0420 to 0.0452 , with an average annual growth rate of $1.52 \%$, that is, the county land use efficiency level and regional differences expanded at the same time. The second stage (20102018): the land use efficiency continued to increase, with an average annual growth rate of $3.76 \%$, and the Theil Index decreased to 0.0353 , and the regional differences of land use efficiency showed a convergence trend.

Table 2. Average land use efficiency value of Lanxi Urban Agglomeration and Theil Index

\begin{tabular}{|c|c|c|c|c|}
\hline & $\mathbf{2 0 0 6}$ & $\mathbf{2 0 1 0}$ & $\mathbf{2 0 1 4}$ & $\mathbf{2 0 1 8}$ \\
\hline AverageLUE & 0.4995 & 0.5484 & 0.6623 & 0.7134 \\
\hline Theil Index & 0.0420 & 0.0452 & 0.0420 & 0.0353 \\
\hline
\end{tabular}

\subsection{Spatial differentiation characteristics of land use efficiency}

\subsubsection{General distribution characteristics}

Using ArcGIS, the land use efficiency values of counties in 2006, 2010, 2014 and 2018 were divided into four categories to discuss the spatial distribution characteristics and laws of land use efficiency (Fig1).

The land use efficiency of counties in Lanxi Urban Agglomeration shows obvious spatial distribution differences, with the pattern characteristics of high center-low periphery, high west and low east, and northsouth differentiation. Among them, Lanzhou and Xining urban district are the regions with the highest land use efficiency in the whole urban agglomeration. In the central region, the land use efficiency is gradually reduced from the center of the Urban Agglomeration to the periphery, which has a significant hierarchical effect. The high-efficiency areas are mainly distributed in a "banded" connection in space, such as Ping'an-Ledu, 
Honggu-Xigu-Anning-Qilihe. The lower-efficiency areas begin to spread to the east and southwest of the urban agglomeration. In 2006, the number of low-efficiency areas was as high as 15 , covering the southern and northeastern regions continuously; In 2018, the lowefficiency areas were transferred to the southeast of urban agglomeration, and the number was reduced to 6 , mainly including Jingtai, Jishishan and Yongjing. Generally speaking, the land use efficiency of most counties in Lanxi Urban Agglomeration has reached high-efficiency and above, and the number of counties in low-efficiency areas has been greatly reduced. The urban agglomeration is further away from realizing the goal of rational layout and coordinated development of land use and production.
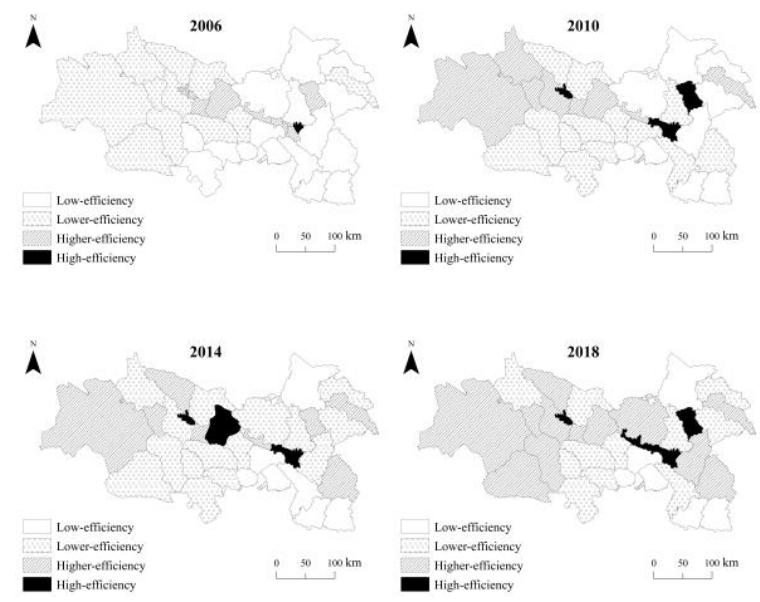

Fig.1. Spatial distribution of land use efficiency from 2006 to 2018

\subsubsection{Differences between the two regions}

From the perspective of regional differences between Gansu and Qinghai, this paper explores the law of spatial differences in land use efficiency at county level. From 2006 to 2018, the land use efficiency of counties in Gansu and Qinghai in Lanxi Urban Agglomeration gradually increased, with the growth rates of $39.11 \%$ and $43.14 \%$ respectively. Qinghai was always higher than Gansu, showing the spatial decreasing law of "west-east". The land use efficiency of counties in Gansu is relatively high in Theil Index and relatively low in Qinghai. The degree of spatial disequilibrium in Gansu and Qinghai is declining, and the difference between regions is higher than that between regions, which indicates that the imbalance of internal development in the two regions restricts the level of land use efficiency. Qinghai has a good ecological environment foundation and high vegetation coverage, so it is necessary to achieve sustainable socio-economic development on the premise of harmonious development between man and nature, with the pressure of ecological environment gradually decreasing and land use efficiency steadily increasing. The proportion of traditional industries with high energy consumption and high pollution in Gansu Province is relatively high, and the industrial structure is unreasonable and low-level, which is unbalanced within the region. With the implementation of ecological civilization construction by Gansu government, land use efficiency began to improve and regional differences narrowed.

\subsection{Spatial correlation characteristics of county land use efficiency}

\subsubsection{Global Spatial Autocorrelation analysis}

The global Spatial Autocorrelation model is used to further understand the spatial agglomeration and evolution characteristics of efficiency in county land use efficiency of Lanxi Urban Agglomeration. From 2006 to 2018, the Global Moran's I was greater than 0 and passed the significance test with $\mathrm{P}$ value less than 0.05 , which indicated that there is a positive spatial correlation between the land use efficiency of counties in Lanxi Urban Agglomeration, that is, the research units with higher efficiency tended to be adjacent, while the research units with lower efficiency were adjacent. Among them, Global Moran's I declined from 2010 to 2018 , but showed an overall increasing trend, indicating that the dynamic change of county land use efficiency level was relatively stable, and the spatial agglomeration situation was weakened, but it still showed strong spatial agglomeration characteristics.

\subsubsection{Local Spatial Autocorrelation analysis}

Global Spatial Autocorrelation can't reflect the specific location of agglomeration or anomaly, but Local Spatial Autocorrelation is needed to reflect the correlation between the attribute values of research units and adjacent units in the study area, and to test the degree of agglomeration and difference between local research units. Combined with LISA cluster diagram of land use efficiency in Lanxi Urban Agglomeration, it is found that:

(1) High-high concentration is mainly distributed in the central cities of Lanzhou and Xining, among which Anning, Chengbei and Chengzhong always belong to this type of area. This type of area belongs to a class of areas with obvious location advantages, high level of economic development and technology, reasonable industrial structure and strict environmental management system in urban agglomeration. The land use efficiency keeps a high level, and it has a positive driving effect on adjacent areas, with significant spatial spillover effect.

(2) Low-high settlement is located in Huangzhong, Gaolan, Huzhu and other county units. The land use efficiency in these areas is far lower than that in the surrounding areas. Although they are distributed in the diffusion effect areas in areas with high land use efficiency, their ability to accept the "diffused" effect is limited, and there is still room for improvement in land use efficiency.

(3) High-low agglomeration is relatively dispersed in space. This kind of region has obvious advantages in 
resource endowment, little negative effects on environment and high level of land use efficiency. However, due to the lack of effective regional cooperation mechanism and unreasonable industrial chain layout, it is difficult to bring radiation to the surrounding areas, which leads to the polarization phenomenon of high land use efficiency and low land use efficiency in the surrounding areas.

(4) Low-low concentration is concentrated in the southern area of Lanxi Urban Agglomeration, which is backward in economic development, fragile in ecological environment, low in resource utilization rate, far away from the radiation range of high-efficiency areas, and low in land use output level. In 2014, the An'ding changed from a low-low gathering area to a high-low gathering area; Longxi, Weiyuan and Lintao changed from low-low agglomeration area to insignificant agglomeration trend, which indicated that the low efficiency of land use began to spread from southeast to southwest.

\section{Conclusion and proposal}

(1) From the time change analysis, the average land use efficiency of Lanxi Urban Agglomeration increased by $42.8 \%$ from 0.4995 in 2006 to 0.7134 in 2018, while Theil Index showed an inverted " $v "$ change track, which made the land use efficiency have the evolution characteristics of two stages. That is, from 2006 to 2010, land use efficiency increased and regional differences expanded; From 2010 to 2018, land use efficiency still increased, but regional differences began to shrink.

(2) From the analysis of total spatial differences, the land use efficiency of Lanxi Urban Agglomeration is characterized by high in the central part, low in the periphery, high in the west and low in the east, and differentiated between the north and the south. The number of low-efficiency areas is greatly reduced, while the number of high-efficiency areas is obviously increased, and the level of county land use efficiency has been greatly improved. From the analysis of regional differences, the land use efficiency in Gansu and Qinghai is on the rise, showing the law of "west-east" spatial decline, and the regional differences are the main reason that affects the unbalanced development of land use efficiency in counties of the two regions.

(3) The Global Moran's I index of county land use efficiency in Lanxi Urban Agglomeration is greater than 0 , which indicates that there is a significant spatial correlation between county land use efficiency. From the evolution characteristics of local spatial pattern, it is found that high land use efficiency and high agglomeration are always concentrated in two regional central cities;During the study of low-low agglomeration, the phenomenon of diffusion from southeast to southwest appeared; The distribution of high-low agglomeration is relatively scattered, while the low-high agglomeration is distributed around the high-level land intensive areas in central China, and the number of the two types of areas is basically unchanged.
The regional difference of land use efficiency under the concept of green development is the result of complex interweaving of many factors, which directly reflects the allocation difference of different participating factors in the process of land use. Based on the analysis of the spatial and temporal differences of land use efficiency in Lanxi Urban Agglomeration, this paper puts forward the following suggestions: First, implement land resources classification management and control according to the carrying capacity of resources and environment and the suitability of land space development; The second is to implement ecological coconstruction and environmental co-governance and promote the protection and restoration of key areas and important ecosystems; The third is to restrict backward industries with serious pollution and large consumption of energy resources. Finally, the land use pattern of green land resources utilization and sustainable and coordinated development of regional economy, society and ecological environment system will be realized.

\section{References}

1. J.Zhao,China's Green Development: Opportunities, Challenges and Innovation Strategies. J. Aca Fro, 19, 80 (2013)

2. B.Verburg, M.Derek, Trajectories of land use change in Europe: a model-based exploration of rural futures.J. L E ,2,25 (2010).

3. A.Brett, D.Neville, Land use efficiency: anticipating future demand for land-sector greenhouse gas emissions abatement and managing trade-offs with agriculture, water, and biodiversity.J. GC,11,21(2015).

4. X.Bao, C.Liu, J.Zhang.,Comprehensive evaluation of urban land use efficiency.J. UP, 4,46(2009).

5. D.Wu, H.Mao,X.Zhang, Evaluation of urban land use efficiency in China. J. JOG, 8, 66(2011).

6. S.Liu,X.Ke,Evolution characteristics of land use efficiency in China's urban agglomerations and ways to improve it. J. UP, 9, 54(2019).

7. J.Wan, X.Chen. Spatial pattern and spillover effect of urban land intensive use efficiency in China from the perspective of land development right. J. EG, 6, 38(2018).

8. C.Li, Estimation of land use efficiency of urban agglomeration in the middle reaches of Yangtze River: realistic mechanism and temporal and spatial differentiation. J. CPRE,12,157(2017).

9. J.Liao,F.Han,.Influence of urbanization on land use efficiency in Changsha-Zhuzhou-Xiangtan region.J. C P, 2, 30,(2010).

10. Y.Zhou,G.Tan, Analysis of the Impact of Local Government Competition on Urban Land Use Efficiency.J. RRD, 3, 118(2017).

11. L.Liang,B.Zhai,Influence of economic agglomeration and industrial structure on urban land use efficiency. J. RRD, 3, 36(2017). 